Ciencia y Salud, Vol. III, No. 2, mayo-agosto, 2019 •ISSN (impreso): 2613-8816 • ISSN (en línea): 2613-8824

DOI: https://doi.org/10.22206/cysa.2019.v3i2.pp43-48

\title{
REACCIONES ADVERSAS A LOS FÁRMACOS ANTITUBERCULOSIS EN PACIENTES DE 0 A 18 AÑOS ATENDIDOS EN LA UNIDAD DE TUBERCULOSIS DEL HOSPITAL INFANTIL DOCTOR ROBERT REID CABRAL, JUNIO - DICIEMBRE 2017 REPÚBLICA DOMINICANA
}

\begin{abstract}
Adverse Reactions to Antitituberculosis Drugs in Patients from 0 to 18 years old attended at the Tuberculosis Unit Of The Doctor Robert Reid

Cabral Children's Hospital, June - December 2017

Dominican Republic
\end{abstract}

\author{
Yanira García-García ${ }^{1 *}$, Elsa Camilo-Pantaleón*, Vicenta Sánchez-Sánchez³
}

Recibido: agosto 12, 2018 • Aprobado: noviembre 22, 2018

Cómo citar: García-García Y, Camilo-Pantaleón E, Sánchez-Sánchez V. Reacciones adversas a los fármacos antituberculosis en pacientes de 0 a 18 años atendidos en la unidad de tuberculosis del Hospital Infantil Doctor Robert Reid Cabral, junio - diciembre 2017, República Dominicana. cysa [Internet]. 26 de julio de 2019 [citado 26 de julio de 2019];3(2): 43-48. Disponible en: https://revistas.intec.edu.do/index.php/cisa/article/view/1477

\section{Resumen}

Introducción: la Tuberculosis infantil representa del 6 al $10 \%$ de los casos de TB, la OMS estimó para el 2017 unos 560 (rango: 380 - 740) casos en menores de 15 años en República Dominicana ${ }^{1}$. De los 122 casos diagnosticados en RD, 53 fueron en nuestro hospital. Los efectos adversos es una de las primeras causas de abandono de tratamiento en TB; los infantes no escapan a esta realidad.

Objetivo: determinar las reacciones adversas a los fármacos antituberculosis en pacientes (RAFA) de 0 a 18 años atendidos en la unidad de tuberculosis del Hospital Infantil Dr. Robert Reid Cabral, junio-diciembre 2017.

\footnotetext{
1. Pediatra, Hospital Infantil Dr. Robert Read Cabral, Santo Domingo, República Dominicana

2. Unidad de Sintomáticos Respiratorios, Hospital Infantil Dr. Robert Read Cabral, Santo Domingo, República Dominicana

3. Médico Ayudante, Hospital Infantil Dr. Robert Read Cabral, Santo Domingo, Rep. Dom.
}

\begin{abstract}
Introduction: Childhood Tuberculosis represents 6 to $10 \%$ of TB cases, WHO estimates about 750 cases in children under 15 in the Dominican Republic ${ }^{1}$. Of the 122 cases diagnosed in RD, 53 were in our hospital. Adverse effects is one of the first causes of treatment abandonment in TB, infants do not escape this reality. Objective: To determine the adverse reactions to antituberculosis drugs in patients (RAFA) from 0 to 18 years old treated in the tuberculosis unit of the Dr. Robert Reid Cabral Children's Hospital, June - December 2017.
\end{abstract}

Methodology: Descriptive and transversal study, retrospective data collection. We reviewed all the records of the tuberculosis unit diagnosed during June-December 2017.

\footnotetext{
Correo-e de los autores:

*yanira.garcia@hotmail.com

** elsacamilo@hotmail.com
} 
Metodología: estudio descriptivo y transversal, con recolección de datos retrospectiva. Revisamos todos los expedientes de la unidad de tuberculosis diagnosticados durante junio-diciembre 2017.

Resultados: de los 29 pacientes con tratamiento anti-TB, $16(55.2 \%)$ presentaron efectos adversos (RAFA), $62 \%$ en la primera fase. El sexo más afectado fue el masculino (51.7\%). La mayor prevalencia en los grupos de 5-9 y 10-14 años con $27.6 \%$, respectivamente. El $62 \%$ de los casos fueron de origen rural. La educación del padre fue desconocida en el $79 \%$ de los casos y de la madre $37.9 \%$. El $89.7 \%$ de los casos con efectos adversos recibieron drogas de primera línea, y el $10.3 \%$ drogas de segunda línea. Los efectos gastrointestinales fueron los más frecuentes; las náuseas en el $17.2 \%$, seguida por los vómitos con un $10.3 \%$ de primera línea. Mientras los que utilizaron segunda línea, la náusea se presentó en $6.9 \%$ y los vómitos en $10.3 \%$. Dentro de los efectos adversos el $93.8 \%$ se clasificaron como leve. El $37 \%$ de los pacientes finalizaron sin complicaciones.

Conclusión: la medicación anti-tuberculosis es segura y los efectos adversos no afectaron la adherencia al tratamiento en el grupo estudiado.

Palabras clave: tuberculosis; infantil; farmacos; tratamiento; reacciones adversas.

\section{Introducción}

La tuberculosis es una enfermedad infecciosa, causada por el Mycobacterium tuberculosis, afecta cualquier órgano de la economía, siendo el más frecuente el pulmón. Se transmite fundamentalmente por vía aerógena, a través de gotículas generadas en el aparato respiratorio de pacientes con enfermedad pulmonar activa. $^{2}$

Esta es una enfermedad que se ha convertido en un grave problema de salud pública a nivel mundial. En América, la notificación de TB se ha mantenido estable en el último quinquenio con una tasa alrededor de 22 casos/100,000 habitantes, con incrementos de los casos de drogorresistencia. En República Dominicana, en los últimos ańos, se ha registrado una disminucion de la tasa de tuberculosis, con
Results: Of the 29 patients with anti-TB treatment, 16 (55.2\%) had adverse effects (RAFA), $62 \%$ in the first phase. The sex most affected was male $(51.7 \%)$. The highest prevalence in the groups of 5-9 and 10-14 years with $27.6 \%$ respectively. $62 \%$ of the cases were of rural origin. The education of the father was unknown in 79 $\%$ and of the mother $37.9 \% .89 .7 \%$ of the cases with adverse effects received first line drugs, and $10.3 \%$ second line drugs. The gastrointestinal effects were the most frequent; nausea in $17.2 \%$, followed by vomiting with 10.3 $\%$ of first line. While those who used the second line, nausea occurred in $6.9 \%$ and vomiting in $10.3 \%$. Among the adverse effects, $93.8 \%$ were classified as mild. $37 \%$ of patients completed treatment successfully, $58 \%$ are in the support phase without complications.

Conclusion: The anti-tuberculosis medication is safe and the adverse effects did not affect the adherence to the treatment in the group studied.

Keywords: Tuberculosis; child; drugs; treatment; adverse reactions.

incremento de los casos de tuberculosis multidrogorresistente (TB-MDR), de coinfección TB-VIH, al igual que en el resto de América ${ }^{3}$.

La terapia de la tuberculosis incluye fármacos orales de primera línea como la Rifampicina (RIF), Isoniazida (INH), Pirazinamida (PZA), Etambutol (EMB); además de los fármacos de segunda línea utilizados en el tratamiento de las drogoresistencia como son los inyectables (Kanamicina, Amikacina, Capreomicina), Cicloserina, Ethionamida y las fluroquinolonas. Las reacciones adversas a fármacos antituberculosos (RAFA) es una de las principales causas de falta de adherencia al tratamiento de estos pacientes, ocasionando resistencia a los fármacos o el abandono del tratamiento en aproximadamente el $8.7 \%$. 
Reacciones adversas a los fármacos antituberculosis en pacientes de 0 a 18 años atendidos en la unidad de tuberculosis del Hospital Infantil Doctor Robert Reid Cabral, junio - diciembre 2017, República Dominicana

Ambas situaciones son de mucha gravedad, el abandono total trae como consecuencia mantener la cadena epidemiológica, no curación de los pacientes y en casos más graves, la muerte del enfermo; en casos de abandono parcial, el riesgo mayor después de la no curación es la creación de resistencia a los fármacos de primera línea. En niños, el tema de las RAFA ha sido poco abordado, esta es la razón por la cual nos planteamos realizar esta investigación.

\section{Materiales}

Se realizó un estudio descriptivo, transversal, de recolección de datos retrospectiva, que pretende determinar las reacciones adversas a los fármacos antituberculosis en pacientes de 0 a 18 años atendidos en el Hospital Infantil Doctor Robert Reid Cabral, Santo Domingo, junio-diciembre 2017. Se revisaron todos los expedientes del archivo de la unidad de tuberculosis durante el periodo antes descrito, donde fueron excluidos los expedientes que no fueron encontrados $y$ aquellos pacientes que tuvieron seguimiento fuera de la unidad. Se procesaron los datos en Microsoft Word, Excel 2007 y SPSS versión 23 . Se realizó un análisis estadístico de frecuencias simples. Los datos recopilados en este estudio fueron manejados con el estricto apego a la confidencialidad.

\section{Resultados}

En la consulta de la Unidad de Tuberculosis Infantil del Hospital Infantil Dr. Robert Reid Cabral, en el periodo de estudio, se encontraron un total de 29 pacientes menores de 18 años de edad con diagnóstico de tuberculosis, los cuales recibieron tratamiento anti-TB, 27 (93\%) con TB drogo sensible y $5(10.3 \%)$ drogo resistente. De ellos, 16 (55.2\%) presentaron efectos adversos (RAFA), $62 \%$ en la primera fase. El sexo más afectado fue el masculino $(51.7 \%)$. La mayor prevalencia en los grupos de 5-9 y 10-14 años con $27.6 \%$, respectivamente. El $62 \%$ de los casos fueron de origen rural. La educación del padre fue desconocida en el $79 \%$ y la de la madre $37.9 \%$. El $89.7 \%$ de los casos con efectos adversos recibieron drogas de primera línea, y el $10.3 \%$ drogas de segunda línea. Los efectos gastrointestinales fueron los más frecuentes; las náuseas en el $17.2 \%$, seguida por los vómitos con un $10.3 \%$ en los pacientes con tratamiento de primera línea. En los pacientes con tratamiento con drogas de segunda línea, la náusea se presentó en $6.9 \%$ y los vómitos en $10.3 \%$.

Dentro de los efectos adversos el $93.8 \%$ se clasificó como leve. El $37 \%$ de los pacientes finalizaron tratamiento de forma exitosa, mientras que el $58 \%$ está en fase de sostén sin complicaciones.

Cuadro 1. Distribución por edad

\begin{tabular}{|c|c|c|c|}
\hline $\begin{array}{c}\text { Edad } \\
\text { (años) }\end{array}$ & Frecuencia & Porcentaje & $\begin{array}{c}\text { Porcentaje } \\
\text { Acumulado }\end{array}$ \\
\hline 1 a 4 & 6 & 20.7 & 20.7 \\
\hline 5 a 9 & 8 & 27.6 & 48.3 \\
\hline 10 a 14 & 8 & 27.6 & 75.9 \\
\hline 15 a 18 & 7 & 24.1 & 100 \\
\hline Total & 29 & 100 & \\
\hline
\end{tabular}

Fuente: elaboración propia.

Cuadro 2. Efectos adversos según condición bacteriológica

\begin{tabular}{|c|c|c|}
\hline $\begin{array}{c}\text { Condición } \\
\text { bacteriológica }\end{array}$ & No. Pacientes & Porcentaje \\
\hline Drogo sensible & 27 & 89.7 \\
\hline Drogo resistente & 3 & 10.3 \\
\hline Total & 29 & 100 \\
\hline
\end{tabular}

Fuente: elaboración propia. 
Cuadro 3. Reacciones adversas a fármacos antituberculosis

\begin{tabular}{|c|c|c|c|c|c|c|c|c|}
\hline \multirow{3}{*}{ Efectos adversos } & \multicolumn{8}{|c|}{ Tratamiento RAFA } \\
\hline & \multicolumn{4}{|c|}{ Fase intensiva } & \multicolumn{4}{|c|}{ Fase de sostén } \\
\hline & $1^{\text {ra }}$ Línea & $\%$ & $2^{\text {da }}$ Línea & $\%$ & $1^{\text {ra }}$ Línea & $\%$ & $2^{\text {da }}$ Línea & $\%$ \\
\hline Náuseas & 5 & 17.2 & 0 & 0 & 2 & 6.9 & 1 & 3.4 \\
\hline Vómitos & 3 & 10.3 & 0 & 0 & 3 & 10.3 & 1 & 3.4 \\
\hline Ictericia & 1 & 3.4 & 0 & 0 & 1 & 3.4 & 0 & 0 \\
\hline Hipoacusia & 0 & 0 & 0 & 0 & 0 & 0 & 0 & 0 \\
\hline Epigastralgia & 1 & 3.4 & 0 & 0 & 1 & 3.4 & 0 & 0 \\
\hline Otros & 1 & 3.4 & 1 & 3.4 & 1 & 3.4 & 0 & 0 \\
\hline
\end{tabular}

Fuente: elaboración propia.

Cuadro 4. Efectos adversos por fase de tratamiento

\begin{tabular}{|c|c|c|c|}
\hline \multirow{2}{*}{$\begin{array}{c}\text { Tratamiento } \\
\text { RAFA }\end{array}$} & Frecuencia & Porcentaje \\
\hline \multirow{2}{*}{ Fase intensiva } & $\begin{array}{c}\text { Primera } \\
\text { Línea }\end{array}$ & 9 & 34.6 \\
\cline { 2 - 4 } & $\begin{array}{c}\text { Segunda } \\
\text { Línea }\end{array}$ & 1 & 33.3 \\
\hline $\begin{array}{c}\text { Primera } \\
\text { Línea }\end{array}$ & 4 & 15.3 \\
\cline { 2 - 4 } & $\begin{array}{c}\text { Segunda } \\
\text { Línea }\end{array}$ & 2 & 66.66 \\
\hline No efectos \\
adversos & 13 & 50 \\
\hline Total & 29 & 100 \\
\hline
\end{tabular}

Fuente: elaboración propia.

Cuadro 5. Clasificación por severidad

\begin{tabular}{|c|c|c|}
\hline Clasificación & Frecuencia & Porcentaje \\
\hline Leve & 15 & 93.8 \\
\hline Moderado & 1 & 6.3 \\
\hline Severa & 0 & 0 \\
\hline Total & 16 & 100 \\
\hline
\end{tabular}

Fuente: elaboración propia.
Cuadro 6. Tratamiento de los efectos adversos

\begin{tabular}{|c|c|c|}
\hline $\begin{array}{c}\text { Manejo de los } \\
\text { efectos adversos }\end{array}$ & Frecuencia & Porcentaje \\
\hline Orientación & 15 & 93.7 \\
\hline Medicación & 1 & 6.3 \\
\hline $\begin{array}{c}\text { Suspensión de } \\
\text { medicación }\end{array}$ & 0 & 0 \\
\hline Total & 16 & 100 \\
\hline
\end{tabular}

Fuente: elaboración propia.

Cuadro 7. Desenlace de los pacientes tratados

\begin{tabular}{|c|c|c|}
\hline $\begin{array}{c}\text { Desenlace por } \\
\text { efectos adversos }\end{array}$ & Frecuencia & Porcentaje \\
\hline Paciente curado & 0 & 0 \\
\hline $\begin{array}{c}\text { Tratamiento } \\
\text { finalizado }\end{array}$ & 6 & 37.9 \\
\hline En tratamiento & 9 & 58.6 \\
\hline $\begin{array}{c}\text { Perdido en } \\
\text { seguimiento }\end{array}$ & 0 & 0 \\
\hline Fallecido & 1 & 3.4 \\
\hline Total & 16 & 100 \\
\hline
\end{tabular}

Fuente: elaboración propia. 
Reacciones adversas a los fármacos antituberculosis en pacientes de 0 a 18 años atendidos en la unidad de tuberculosis del Hospital Infantil Doctor Robert Reid Cabral, junio - diciembre 2017, República Dominicana

\section{Discusión}

La tuberculosis es una enfermedad bacteriana crónica causada por el mycobacterium tuberculosis, que se caracteriza por la formación de granuloma en los tejidos infectados y una hipersensibilidad mediada por células, generalmente localizada en los pulmones, también puede afectar cualquier otro órgano. ${ }^{5}$

Esta situación se reproduce en países en vías de desarrollo; de forma particular en República Dominicana, en donde la tuberculosis continúa siendo un importante problema de salud pública, debido a que no se observa una tendencia a la resolución espontánea de la enfermedad sin recurrir a programas de control rigurosos, como los que se llevan actualmente en los centros regionales de salud y en las unidades de atención primaria, también llamadas clínicas rurales.

A nivel internacional, la incidencia de RAFA en cuanto al género es ligeramente superior en el sexo femenino. En esta serie el sexo más afectado fue el masculino con un $51.7 \%$. Es conocido que las RAFA se presentan con más frecuencia en la fase intensiva del tratamiento, sobre todo en las primeras semanas de iniciar la medicación. Díaz reporta mayor incidencia de RAFA's en la fase intensiva con el $31.0 \%$ de los casos ${ }^{6}$. El $89.7 \%$ (26) de los casos con efectos adversos recibieron drogas de primera línea, y el $10.3 \%$ (3) drogas de segunda línea. Es importante destacar que los tres pacientes en tratamiento con drogas de segunda línea presentaron efectos adversos, uno en la primera fase y dos en la fase de sostén. Los medicamentos de segunda línea son más tóxicos y el tratamiento es más prolongado, por lo que se esperan mayores efectos adversos. ${ }^{6}$

Los efectos adversos sobre el sistema gastrointestinal son los RAFA más frecuente en el tratamiento anti TB de primera y segunda línea. La fiebre, que puede ser considerada parte de la enfermedad o efecto adverso, se presentó en un $10.3 \%$ en la fase intensiva y un $6.9 \%$ en la fase de sostén, en aquellos pacientes que llevaron la primera línea; mientras que los que recibieron segunda, la fiebre no se presentó en ninguna de las fases del tratamiento. ${ }^{3-6}$

Las RAFA se clasifican por su severidad en leve y severa, dentro de los efectos adversos encontrados el $93.8 \%$ se clasificaron como leves, los cuales se manejaron con orientación a los padres. A un caso de TB drogorresistente, manejado con drogas de segunda línea, se le suministró protectores gástricos. En ninguno de los casos se suspendió medicación. De los tres (3) pacientes en tratamiento con drogas de segunda línea se les realizó audiometría a dos de ellos, resultando normal. Los efectos adversos en los pacientes seguidos son similares a lo publicado en otras series de pacientes con tuberculosis infantil.

En el desenlace de los pacientes con diagnóstico de tuberculosis que presentaron efectos adversos encontramos que $37 \%$ de los pacientes finalizaron tratamiento de forma exitosa, el $58 \%$ está en fase de sostén sin complicaciones; un paciente fallecido, por causa no relacionada al tratamiento.

\section{Conclusión}

Más de la mitad de los pacientes que recibieron tratamiento anti-tuberculosis de primera o segunda línea en el período de estudio presentaron efectos adversos leves o moderados. Siendo más frecuente en la fase intensiva.

La medicación anti-tuberculosis es segura y los efectos adversos no afectaron la adherencia al tratamiento en el grupo de paciente seguido.

\section{Bibliografía}

1. World Health Organization (OMS). Global Tuberculosis Report 2017. Washinthon: OMS; 2017.

2. Organización Mundial de la Salud (OMS). Temas de salud: Tuberculosis. [Online]. Available from: http://www.who.int/topics/ tuberculosis/es/ [Accessed 24 $4^{\text {th }}$ november 2016]. 
3. Organización Panamericana de la Salud (OPS). Situacion dela tuberculosis en las Américas 2016. [Online]. Available from: PShttps://www.paho. org/hq/dmdocuments/2017/2017-cha-hoja-info-situacion-tb-americas.pdf. [Accessed 25th may 2018].

4. Victoriano F, Caminero J. Tuberculosis. (3ra ed.) Santiago de Chile: Editorial Mediterráneo; 2011.

5. Navarro C, Mendoza JL. Factores asociados al abandono del tratamiento antituberculoso en paciente con TB. Ciencia y Cuidado. 2013;10(1): 19-27. ISSN 1794-9831.

6. Díaz Covarrubias-López T, Laniado-Laborín R. Reacciones adversas a los fármacos antituberculosis en pacientes con esquemas mixtos. Neumol. cir. torax [Internet]. 2016 Jun [citado 10 de octubre 2017];75( 2 ): 149-154. Available from: http://www.scielo.org.mx.

7. Isselbacher K, Brauwald E, Wilson J, Martin J, Fauci A, Kasper D. Principios de Medicina Interna por Harrison.(13th ed.). Madrid: Mc.Graw Hill; 2005.

8. Bisero E, Luque G, Framarin G, Reacciones adversas a fármacos antituberculosos en pediatría. A propósito de 4 hermanos. Revista Americana de Medicina Respiratoria. 2016;16(3): 273-8 Available from: http://www.scielo.org.ar/pdf/ ramer/v16n3/v16n3a10.pdf.

9. Mamani-Poma A. Reacción adversa a fármacos antituberculosos (RAFA). Una realidad en Bolivia. J. Selva Andina Res. Soc. [Internet]. 2018 [citado 30 de mayo 2018 ];9(1): 62-63. Available from: http://www.scielo.org.bo/scielo.php?script=sci_ arttext\&pid=S2072-92942018000100006\& $\operatorname{lng}=\mathrm{es}$.
10. López López A, Hernández Coronado P. Reacciones adversas a fármacos antituberculosos en hospitales de III nivel, Caja Nacional de Salud-Hospital Viedma abril 2002 a abril 2005. Rev. méd. (Cochabamba) [Internet]. 2007 Ago [citado 20 de mayo 2018];18(28): 32-41. Available from:http://www.revistasbolivianas.org.bo/scielo.php?script=sci_arttext \&pid=S2074-46092007002800006\&lng=es.

11. Aguayo M, Rodríguez JC. Hepatotoxicity of antituberculosis therapy. Rev. chil. enferm. respir. [Internet]. 2011 Mar [citado 20 de mayo 2018];27(1): 53-7. doi: http://dx.doi. org/10.4067/S0717-73482011000100010.

12. Ministerio de Salud Pública. Guía nacional para el diagnóstico, tratamiento y prevención de tuberculosis en niños, niñas y adolescentes. Santo Domingo: MISPAS; 2012.

13. Ministerio de Salud Pública. Reglamento técnico para la prevención y el control de la tuberculosis. Santo Domingo: MISPAS; 2014.

14. Ramos Amador JT, Illán Ramos M, Francisco González L. Actualización en tuberculosis infantil. En: AEPap (ed.). Curso de Actualización Pediatría 2018. Madrid: Lúa Ediciones 3.0; 2018. p. 253-257.

15. Mellado Peńa M.J, et al. Actualización del tratamiento de la tuberculosis en niños. An Pediatr (Barc). 2018;88(1): 52. 